\title{
A novel description of ISFET sensitivity with the buffer capacity and double-layer capacitance as key parameters
}

\author{
R.E.G. van Hal, J.C.T. Eijkel, P. Bergveld \\ MESA Research Institute, University of Twente, PO Bax 217, 7500 AE Enschede, Netheriands
}

\begin{abstract}
The $\mathrm{pH}$ sensitivity of ISFETs arises from interactions of protons with ISFET gate surface sites. This sensitivity is described by a new simpler model with the intrinsic buffer capacity and the differential capacitance as key parameters. The obtained expression is independent of the models used for the chemical surface equilibria and the charge profile in the solution. The general expression for the sensitivity is elaborated using the site-binding theory and the Gouy-Chapman-Stern theory. The relatively high sensitivity of $\mathrm{Ta}_{2} \mathrm{O}_{5}$ ISFETs is explained using this elaborated theory. It is shown that the electrolyte concentration has almost no influence on the sensitivity of $\mathrm{Ta}_{2} \mathrm{O}_{5}$ ISFETs.
\end{abstract}

Keywords: Buffer capacity; Double-layer capacitance; ISFETs

\section{Introduction}

Shortly after the introduction of the ISFET, it was noticed that these devices were sensitive to $\mathrm{pH}$ [1]. The operational mechanism of the ISFET is described by Bergveld and Sibbald [2] as an expression for the drain current, $I_{\mathrm{D}}$, in the unsaturated region:

$$
\begin{aligned}
I_{\mathrm{D}}= & \mu C_{\mathrm{ox}} \frac{W}{L}\left\{\left[V_{\mathrm{GS}}-\left(E_{\mathrm{ref}}-\psi_{0}+\chi^{\mathrm{sol}}-\frac{\Phi_{\mathrm{Si}}}{q}\right.\right.\right. \\
& \left.\left.\left.-\frac{Q_{\mathrm{ox}}+Q_{\mathrm{ss}}}{C_{\mathrm{ox}}}-\frac{Q_{\mathrm{B}}}{C_{\mathrm{ox}}}+2 \phi_{\mathrm{f}}\right)\right] V_{\mathrm{DS}}-1 / 2 V_{\mathrm{DS}}^{2}\right\}
\end{aligned}
$$

where $\mu$ is the average electron mobility in the channel; $W$ and $L$ are respectively the width and the length of the gate; $E_{\text {ref }}$ is the contribution of the reference electrode; $V_{D S}^{r}$ and $V_{G S}$ are respectively the drain-source voltage and the gate-source voltage; $\Phi_{\mathrm{si}}$ is the silicon electron work function; $q$ is the elementary charge; $C_{\mathrm{ox}}$ is the capacitance of the gate oxide; $Q_{\mathrm{ox}}, Q_{\mathrm{ss}}$ and $Q_{\mathrm{B}}$ are the charges located in the oxide, charges located in surface states and interface states and the depletion charge respectively; $\chi^{\text {sol }}$ is the surface dipole potential of the solution; $\phi_{\mathrm{f}}$ is the potential difference between the Fermi levels of doped and intrinsic silicon. All parameters are constant except the electrostatic po- tential at the surface, $\psi_{0}$, and the surface dipole potential. The surface dipole potential is supposed to be independent of $\mathrm{pH}$. Therefore changes in the drain current are attributed to changes in the electrostatic potential, $\psi_{0}$, only.

From the observed short response times and the sensitivity, which was below $59.2 \mathrm{mV}$ for $\mathrm{SiO}_{2}$, it was concluded that surface reactions between the gate insulator and the electrolyte should determine the primary response mechanism [3]. In 1974 Yates et al. [4] introduced the site-binding model in colloid chemistry to describe the properties of an oxide-aqueous electrolyte interface. This model was later adapted to describe the insulator-electrolyte interface of an ISFE'I. Nowadays, the model presented by Bousse [5] is commonly accepted as a good description for the ISFET response. However, the model is not able to give a description of the sensitivity that can easily be interpreted. Moreover, the model is not valid over the entire $\mathrm{pH}$ range and the influence of the ionic strength is neglected.

In this paper a simpler theory is presented that is valid over the entire $\mathrm{pH}$ range. This theory is derived using the site-binding model, but is valid for all theories that describe the charging of an insulator-electrolyte 
interface as a result of interactions that take place at specific sites at the surface.

\section{The pH sensitivity of ISFETs}

In this section a general expression for the $\mathrm{pH}$ sensitivity, which is the change of the insulator-electrolyte potential, $\psi_{0}$, on a change of the bulk $\mathrm{pII}, \delta \psi_{0} / \delta \mathrm{pH}_{\mathrm{B}}$, is given. This expression is derived from a separate treatment of both sides of the double layer, i.e., the gate insulator and the electrolyte.

The site-binding model describes the charging mechanism of an oxide as the equilibrium between the $\mathrm{AOH}$ surface sites and the $\mathrm{H}^{+}$ions in the bulk of the solution. The surface reactions are [4]

$$
\begin{aligned}
& \mathrm{AOH} \rightleftharpoons \mathrm{AO}^{-}+\mathrm{H}_{\mathrm{B}}^{+} \\
& \text {and } \mathrm{AOH}_{2}{ }^{+} \rightleftharpoons \mathrm{AOH}+\mathrm{H}_{\mathrm{B}}{ }^{+}
\end{aligned}
$$

where $B$ refers to the bulk. The equilibrium conditions are

$\frac{\nu_{\mathrm{AO}-} a_{\mathrm{Hs}^{+}}}{\nu_{\mathrm{AOH}}}=K_{\mathrm{a}}$ and $\frac{\nu_{\mathrm{AOH}} a_{\mathrm{Hs}^{+}}}{\nu_{\mathrm{AOH}_{2}+}}=K_{\mathrm{b}}$

where the $K$ values are dimensionless dissociation constants; $\nu_{i}$ is the number of sites per unit area and $a_{\mathrm{Hs}^{+}}$is the activity of $\mathrm{H}^{+}$directly at the insulator surface, related to the bulk activity, $a_{\mathrm{HB}^{+}}$, by the Nernst equation:

$a_{\mathrm{HS}^{+}}=a_{\mathrm{HB}^{+}} \exp \left(-q \psi_{\mathrm{o}^{\prime}} / k T\right)$

where $q$ is the elementary charge, $k$ is the Boltzmann constant and $T$ is the absolute temperature. The surface charge density, $\sigma_{0}$, is given by

$\sigma_{0}=q\left(\nu_{\mathrm{AOH}_{2}+}-\nu_{\mathrm{AO}^{-}}\right)=q N_{\mathrm{S}}\left(\Theta^{+}-\Theta^{-}\right)$

where $N_{\mathrm{s}}$ is the number of sites per unit area; $\Theta^{+}$and $\Theta$ are the fractions of $N_{\mathrm{s}}$ carrying charge, i.e., $\mathrm{AOH}_{2}{ }^{+}$ and $\mathrm{AO}^{-}$, respectively. The fractions $\Theta^{+}$and $\Theta^{-}$are calculated from the cquilibrium rcactions and substituted in Eq. (5) to give

$\sigma_{0}=q N_{\mathrm{S}}\left(\frac{a_{\mathrm{HS}^{+}}^{2}-K_{\mathrm{a}} K_{\mathrm{b}}}{K_{\mathrm{a}} K_{\mathrm{b}}+K_{\mathrm{b}} a_{\mathrm{HS}^{+}}+a_{\mathrm{HS}^{+}}^{2}}\right)=-q[\mathrm{~B}]$

where $[\mathrm{B}]$ is the number of negatively charged groups minus the number of positively charged groups per unit area. $\mathrm{pH}_{\mathrm{pzc}}, \mathrm{pH}$ at the point of zero charge, is defined as the $\mathrm{pH}$ were both fractions are equal and $[\mathrm{B}]$ is zero. The change in the number of charged groups as a result of an infinitesimal increase in $\mathrm{pH}_{\mathrm{S}}$ is the intrinsic buffer capacity, $\beta_{\text {in } t \text { : }}$ :

$\frac{\delta \sigma_{0}}{\delta \mathrm{pH}}=-q \frac{\delta[\mathbf{B}]}{\delta \mathrm{p} \mathrm{H}_{\mathrm{S}}}$

$$
\begin{aligned}
& =-q N_{\mathrm{s}} \frac{K_{\mathrm{b}} a_{\mathrm{Hs}^{+}}^{2}+4 K_{\mathrm{a}} K_{\mathrm{b}} a_{\mathrm{Hs}^{+}}+K_{\mathrm{a}} K_{\mathrm{b}}^{2}}{\left(K_{\mathrm{a}} K_{\mathrm{b}}+K_{\mathrm{b}} a_{\mathrm{HS}^{+}}+a_{\mathrm{H}^{+}}^{2}\right)^{2}} 2.3 a_{\mathrm{Hs}^{+}} \\
& =-q \beta_{\mathrm{int}}
\end{aligned}
$$

The charge in the electrolyte is equal but opposite to the charge on the oxide surface. The Gouy-Chapman-Stern model is used to describe the electrolyte side of the double layer. This model involves a diffuse layer of charge in the solution starting at a distance $x_{2}$ from the surface. This distance $x_{2}$ is the plane of closest approach for the centres of the ions in the solution (Stern layer). The charge in the diffuse layer is [6]

$\sigma_{\mathrm{DL}}=-\left(8 k T \epsilon \epsilon_{0} n^{0}\right)^{1 / 2} \sinh \left(\frac{z q \phi_{2}}{2 k T}\right)=-C_{\mathrm{i}} \psi_{0}=-\sigma_{0}$

where $\epsilon_{0}$ is the permittivity of free space and $\epsilon$ is the relative permittivity; $\phi_{2}$ is the potential at $x_{2} ; n^{0}$ is the number concentration of each ion in the bulk and $z$ is the magnitude of the charge on the ions. The integral capacitance, $C_{\mathrm{i}}$, is often denoted as $K$. The ability of the electrolyte to store charge in response to a change in the electrostatic potential is the differential capacitance [6]:

$$
\begin{aligned}
\frac{\delta \sigma_{\mathrm{DL}}}{\delta \psi_{0}} & =-\frac{\delta \sigma_{0}}{\delta \psi_{0}} \\
& =-\frac{\left(2 \epsilon \epsilon_{0} z^{2} q^{2} n^{0} / k T\right)^{1 / 2} \cosh \left(z q \phi_{2} / 2 k T\right)}{1+\left(x_{2} / \epsilon \epsilon_{0}\right)\left(2 \epsilon \epsilon_{0} z^{2} q^{2} n^{0} / k T\right)^{1 / 2} \cosh \left(z q \phi_{2} / 2 k T\right)} \\
& =-C_{\text {dif }}
\end{aligned}
$$

Combination of both sides of the double layer (Eqs. (7) and (9)) yields

$\frac{\delta \mathrm{t} \%_{0}}{\delta \mathrm{pH}}=\frac{\delta \psi_{0}}{\delta \sigma_{0}} \frac{\delta \sigma_{0}}{\delta \mathrm{pH}_{\mathrm{S}}}=\frac{-q \beta_{\text {int }}}{C_{\mathrm{dif}}}$

The relation between $\mathrm{pH}_{\mathrm{S}}$ and $\mathrm{pH}_{\mathrm{B}}$ is given by the Nernst equation (Eq. (4)). Substitution of Eq. (4) in Eq. (10) and rearrangement gives the general expression for the sensitivity of the electrostatic potential to changes in the bulk $\mathrm{pH}$ :

$\frac{\delta \psi_{0}}{\delta \mathrm{pH}_{\mathrm{B}}}=-2.3 \frac{k T}{q} \alpha$

with $\alpha-\frac{1}{\left(2.3 k T C_{\mathrm{dif}} / q^{2} \beta_{\text {int }}\right)+1}$

where $\alpha$ is a dimensionless sensitivity parameter. The value of $\alpha$ varies between 0 and 1 depending on the intrinsic buffer capacity and the differential capacitance. The site-binding theory and the Gouy-Chapman-Stern model were used in the derivation of this model, but other theories can be used as well to determine the intrinsic buffer capacity [7], the differential capacitance and thus the sensitivity parameter $\alpha$. 


\section{Calculation of the sensitivity}

The previous section showed that the sensitivity of the electrostatic potential to changes in $\mathrm{pH}_{\mathrm{B}}$ is related to the intrinsic buffer capacity and the differential capacitance. In this section both parameters will be calculated using the site-binding model to calculate the intrinsic buffer capacity (Eq. (6)) and the GouyChapman-Stern model to calculate the differential capacitance (Eq. (8)). From the corresponding values the sensitivity is calculated (Eq. (10)).

Table 1 shows the literature values for $\mathrm{p} K_{\mathrm{a}}, \mathrm{p} K_{\mathrm{b}}$ and $N_{\mathrm{s}}$ used to calculate the theoretical sensitivity of several oxides. Figs. 1-3 show respectively the intrinsic buffer capacity, the differential capacitance and the sensitivity as functions of $\Delta \mathrm{pH}$ in a $0.1 \mathrm{M}$ electrolyte with a Stern capacitance of $0.2 \mathrm{~F} \mathrm{~m}^{-2} . \Delta \mathrm{pH}$ is the difference between $\mathrm{pH}_{\mathrm{B}}$ and $\mathrm{pH}_{\mathrm{pzc}}$. Fig. 1 shows large variations in the intrinsic buffer capacity between the three oxides. Fig. 2 shows almost no variation in the differential capac-

Table 1

Literature values of several oxide constants

\begin{tabular}{lrrrll}
\hline & $\mathrm{pK} K_{\mathrm{u}}$ & $\mathrm{p} K_{\mathrm{b}}$ & \multicolumn{1}{l}{$N_{\mathrm{S}}$} & $\mathrm{pH}_{\mathrm{p} x \mathrm{c}}$ & Keference \\
\hline $\mathrm{SiO}_{2}$ & 6 & -2 & $5 \times 10^{18}$ & 2 & {$[5]$} \\
$\mathrm{Ai}_{2} \mathrm{O}_{3}$ & 10 & 6 & $8 \times 10^{18}$ & 8 & {$[5]$} \\
$\mathrm{Ta}_{2} \mathrm{O}_{5}$ & 4 & 2 & $10 \times 10^{18}$ & 3 & {$[8]$} \\
\hline
\end{tabular}

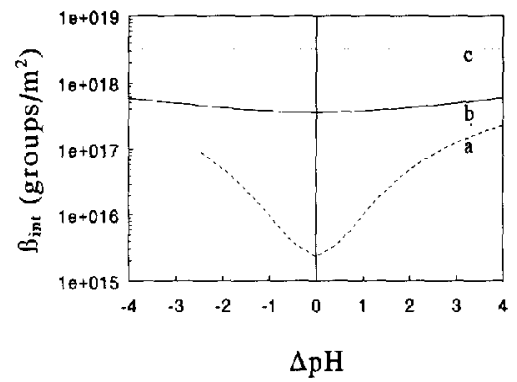

Fig. 1. Cumparison of the theoretical intrinsic buffer capacities of (a) $\mathrm{SiO}_{2}$, (b) $\mathrm{Al}_{2} \mathrm{O}_{3}$, (c) $\mathrm{Ta}_{2} \mathrm{O}_{5}$ (Eq. (7)).

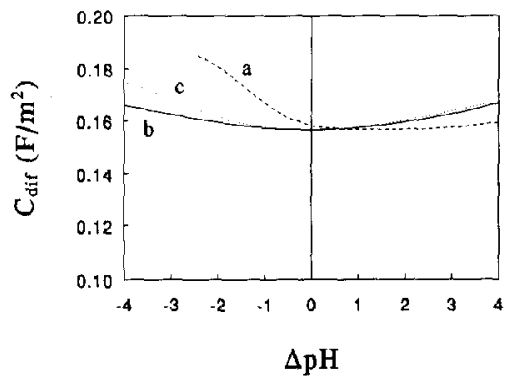

Fig. 2. Comparison of the theoretical differential capacitances for (a) $\mathrm{SiO}_{2}$, (b) $\mathrm{Al}_{2} \mathrm{O}_{3}$, (c) $\mathrm{Ta}_{2} \mathrm{O}_{5}$ (Eq. (9)).

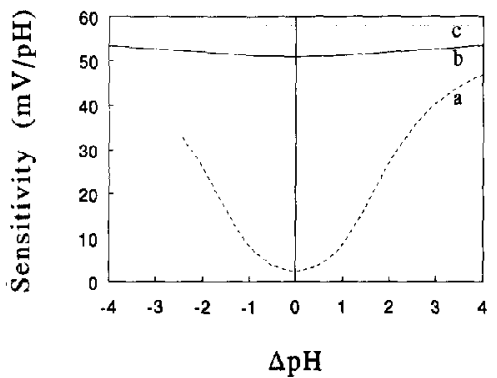

Fig. 3. Comparison of the theoretical sensitivities of (a) $\mathrm{SiO}_{2}$, (b) $\mathrm{Al}_{2} \mathrm{O}_{3}$, (c) $\mathrm{Ta}_{2} \mathrm{O}_{5}$ (Eq. (11))

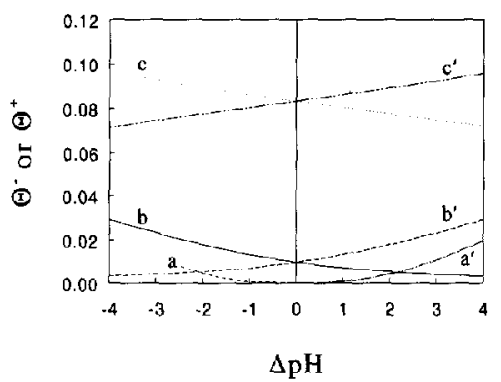

Fig. 4. Fractions of negatively (without prime) and positively (with prime) charged groups of (a) $\mathrm{SiO}_{2}$, (b) $\mathrm{Al}_{2} \mathrm{O}_{3}$, (c) $\mathrm{Ta}_{2} \mathrm{O}_{5}$ (Eqs. (5) and (9))

itance, especially near the point of zero charge. In Fig. 3 , it is shown that the largest variations in sensitivity are at or near the point of zero charge. Thus, it can be concluded that the intrinsic buffer capacity is the major parameter influencing the sensitivity. The intrinsic buffer capacity should be high to give a sensitivity close to the theoretical maximum of $59.2 \mathrm{mV} \mathrm{pH}^{-1}$.

A high buffer capacity can be achieved when the amount of surface sites is high, as follows directly from Eq. (7), or as $\Delta \mathrm{p} K\left(\mathrm{p} K_{\mathrm{b}}-\mathrm{p} K_{\mathrm{a}}\right)$ is small. A small $\Delta \mathrm{p} K$ means that there is a relatively large number of charged groups around the point of zero charge. This does not follow immediately from $\mathrm{Eq}$. (7) but is illustrated by Fig. 4, where the fractions of charged groups are given as a function of $\Delta \mathrm{pH}$. The calculated sensitivities are in good agreement with measured sensitivities [4].

\section{Influence of the ionic strength on the sensitivity of $\mathrm{Ta}_{2} \mathrm{O}_{5}$ ISFETs}

Van Kerkhof et al. showed that $\mathrm{Ta}_{2} \mathrm{O}_{5}$ ISFETs give a fact response to a stepwise change in the ionic strength [9]. The electrostatic potential returns to its original value, in agreement with the observation that the influence of the ionic strength on the static response of an $\mathrm{Ta}_{2} \mathrm{O}_{5}$ ISFET is negligible [10]. The response to stepwise changes in the ionic strength is explained [9] 
by the change in the integral capacitance (Eq. (8)), which is concentration dependent. From Eq. (9) it follows that the ionic strength also directly influences the differential capacitance. Fig. 5 shows the differential capacitance as a function of $\mathrm{pH}_{\mathrm{B}}$ for several ionic strengths using the oxide constants given in Table 1 and a Stern capacitance of $0.2 \mathrm{~F} \mathrm{~m}^{-2}$.

Fig. 5 shows that the differential capacitance can vary up to $50 \%$ around the point of zero charge. A smaller value for the differential capacitance (Fig. 5(c)) causes smaller changes in $\mathrm{pH}_{\mathrm{S}}$ around the point of zero charge (Fig. 6(c)). However, due to this relatively small change in $\mathrm{pH}_{5}$, the intrinsic buffer capacity rises more slowly, as can be seen in Fig. 7(c). These smaller values for the intrinsic buffer capacity partly compensate the

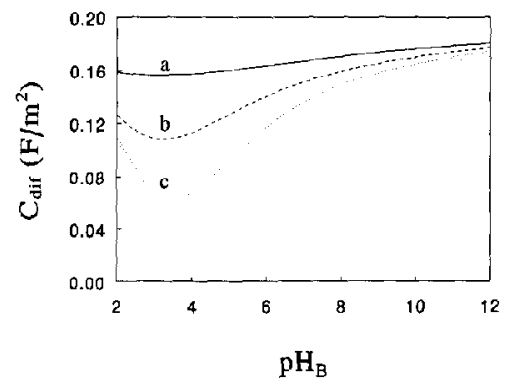

Fig. 5. The differential capacitance of $\mathrm{Ta}_{2} \mathrm{O}_{5}$ ISFETs for (a) $0.1 \mathrm{M}$, (b) $0.01 \mathrm{M}$ and (c) $0.001 \mathrm{M}$ electrolyte solutions (Eq. (9)).

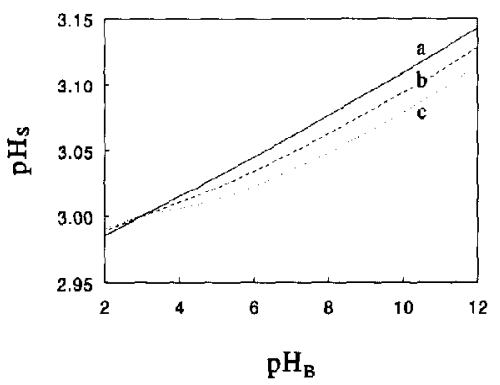

Fig. 6. The $\mathrm{pH}$ at the $\mathrm{Ta}_{2} \mathrm{O}_{5}$ surface as a function of the bulk $\mathrm{pH}$ in (a) $0.1 \mathrm{M}$, (b) $0.01 \mathrm{M}$ and (c) $0.001 \mathrm{M}$ electrolyte solutions.

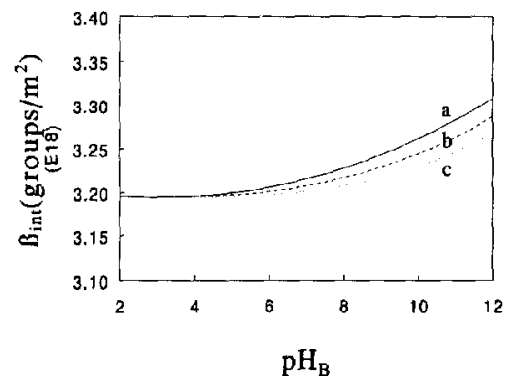

Fig. 7. The intrinsic buffer capacity of a $\mathrm{Ta}_{2} \mathrm{O}_{5}$ ISFET in (a) 0.1 $\mathrm{M}$, (b) $0.01 \mathrm{M}$ and (c) $0.001 \mathrm{M}$ electrolyte solutions (Eq. (7)).

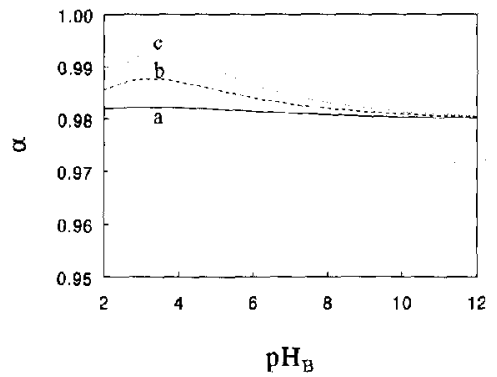

Fig. 8. The theoretical sensitivity parameter for $\mathrm{Ta}_{2} \mathrm{O}_{5}$ in (a) $0.1 \mathrm{M}$, (b) $0.01 \mathrm{M}$ and (c) $0.001 \mathrm{M}$ electrolyte solutions (Eq. (11)).

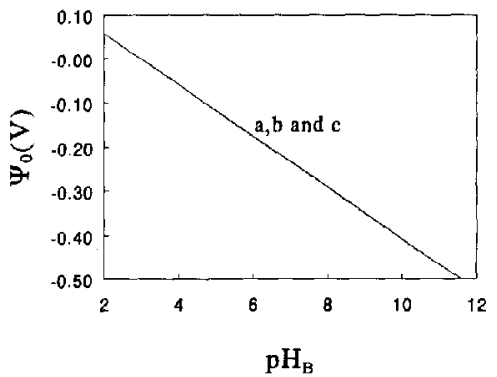

Fig. 9. The theoretical electrostatic potential of $\mathrm{Ta}_{2} \mathrm{O}_{5}$ ISFETs as a function of $\mathrm{pH}$ in (a) $0.1 \mathrm{M}$, (b) $0.01 \mathrm{M}$ and (c) $0.001 \mathrm{M}$ electrolyte solutions (Eqs. (6) and (8))

influence of the ionic strength. The variations in the sensitivity parameter up to $1 \%$ (Fig. 8 ) are therefore smaller than expected from the variations in the differential capacitance (Fig. 5). The theoretical sensitivity at $\mathrm{pH} 12$ is even the same for all calculated concentrations. In Fig. 9 it is clearly shown that the electrostatic potential as measured by the ISFET is negligibly influenced by the ionic strength.

The same compensating mechanism can be expected for other oxides. However, due to the relatively low intrinsic buffer capacity of $\mathrm{Al}_{2} \mathrm{O}_{3}$ compared with $\mathrm{Ta}_{2} \mathrm{O}_{5}$ (Fig. 1), a larger influence on the sensitivity of the electrostatic potential for $\mathrm{pH}_{\mathrm{B}}$ can be expected. This smaller sensitivity was indeed found by Bergveld et al. $[10]$.

\section{Conclusions}

A new general expression for the $\mathrm{pH}$ sensitivity of ISFETs is derived. This expression can be used for all theories that describe the charging of an oxideelectrolyte interface as a result of specific interactions that take place at specific sites. The sensitivity is described in terms of the intrinsic buffer capacity and the differential capacitance. It is shown that a high buffer capacity is necessary for a high sensitivity, which can be achieved by using an oxide with a large amount 
of surface sites as well as by using oxides with a small $\Delta \mathrm{pK}$. Furthermore, it is shown that a high intrinsic buffer capacity minimizes the influence of the electrolyte concentration on the electrostatic potential as measured by the ISFET.

\section{Acknowledgements}

This research was financially supported by the Technology Foundation of the Netherlands (STW) and the Dutch Foundation for Fundamental Research on Matter (FOM).

\section{References}

[1] P. Bergveld, Development of an ion-sensitive solid-state device for neurophysiological measurements, IEEE Trans. Biomed. Eng., BME-17 (1970) 70-71.

[2] P. Bergveld and A. Sibbald, Analytical and Biomedical Applications of ISFETs, Elsevier, Amsterdam, 1988.
[3] W.M. Sui and R.S.C. Cobbold, Basic properties of the electrolyte-SiO-Si $i_{2}$ system: physical and theoretical aspects, IEEE Trans. Electron Devices, ED-26 (1979) 1805-1815.

[4] D.E. Yates, S. Levine and T.W. Healy, Site-binding model of the electrical double layer at the oxide/water interface, J. Chem. Soc. Faraday Trans., 70 (1974) 1807-1818.

[5] L. Bousse, Ph.D. Thesis, Technische Hogeschool Enschede, The Netherlands (1982).

[6] A.J. Bard and L.R. Faulkner, Electrochemical Methods Fundamentals and Applications, Wiley, New York, 1980.

[7] T. Hiemstra, W.H. van Riemsdijk and G.H. Bolt, Multisite proton adsorption modelling at the solid/solution interface of (hydr)oxides: a new approach, J. Colloid Interface Sci., 133 (1989) 91-104.

[8] L. Bousse, S. Mostarshed, B. van der Schoot, N.F. de Rooij, P. Gimmel and W. Göpel, Zeta potential measurements of $\mathrm{Ta}_{2} \mathrm{O}_{5}$ and $\mathrm{SiO}_{2}$ thin films, J. Colloid Interface Sci., 147 (1991) 22-32.

[9] J.C. van Kerkhof, J.C.T. Eijkcl and P. Bergveld, ISFET responses on a stepwise change in electrolyte concentration at constant $\mathrm{pH}$, Sensors and Actuators B, 18-19 (1994) 56-59.

[10] P. Bergycld, R.E.G. van Hal and J.C.T. Eijkel, The remarkable similarity between the acid-base properties of ISFETs and proteins and the consequences for the design of ISFET biosensors, Biosensors Bioelectron., accepted for publication. 